\title{
ANÁLISE DO IMPACTO DA PRODUÇÃO TÉCNICO-CIENTÍFICA DE UMA UNIDADE DA EMBRAPA ESPECIALIZADA EM GEOINFORMAÇÃO
}

\begin{abstract}
Resumo: Um grande desafio imposto às organizações é o de saber transformar, compreender, interpretar e utilizar a enorme massa documental e informacional disponível. No contexto da Embrapa Territorial, as técnicas oriundas da Bibliometria e Cientometria vêm sendo empregadas pela área de Transferência de Tecnologias (TT), para auxiliar no processo de gestão da informação, o qual visa identificar oportunidades a partir do acompanhamento do impacto dos trabalhos produzidos por este centro. Este estudo tem o objetivo de fazer inferências sobre o direcionamento da produção científica da Embrapa Territorial a partir da análise do comportamento dos artigos de periódicos publicados pela instituição no período de 2010 a 2015 . As questões a serem respondidas para alcançar esse objetivo são: 1 - Qual é o quantitativo informacional produzido pela Embrapa Territorial? 1.1. Qual é a qualificação dos periódicos nos quais foram publicados os artigos?; 2 - Qual é o impacto dos trabalhos produzidos pela Unidade nas bases de dados Web of Science e Google Scholar?; 3. Qual é a relação do impacto dos trabalhos com a rede de parcerias da Unidade?
\end{abstract}

Palavras-chave: Produção técnico-científica. Análise métrica. Transferência de tecnologias. Embrapa Territorial.
Daniela Maciel Pinto

Embrapa Territorial Mestra em Ciência da Informação pela Universidade de São Paulo daniela.maciel@embrapa.br

Viviane de Oliveira Solano Embrapa Pantanal

Mestra em Ciência da Informação da Universidade Federal de Minas Gerais viviane.solano@embrapa.br

Celina Maki Takemura Embrapa Territorial Doutora em Ciências da Computação pela

Universidade de São Paulo celina.takemura@embrapa.br

Vera Viana dos Santos Brandão Embrapa Territorial Pós-graduada em Gestão de Unidades de Informação pela Unicep vera.viana@embrapa.br

\section{ANALYSIS OF THE IMPACT OF THE TECHNICAL-SCIENTIFIC PRODUCTION OF AN EMBRAPA UNIT SPECIALIZING IN GEO-INFORMATION}

\begin{abstract}
A great challenge for all organizations is to know how to transform, understand, interpret and use the enormous document and information mass available. In the context of Embrapa Territorial, Bibliometrics and Scientometrics techniques have been employed by the Technology Transfer (TT) area to assist in the information management process, which supports the identification of opportunities based on monitoring the impact of the works produced by the company. This study aims to make inferences about Embrapa Territorial's scientific production by analyzing the behavior of journal articles published by the institution's researchers from 2010 to 2015 . The questions to be answered to achieve this goal are: 1 - What is the amount of information produced by Embrapa Territorial? 1.1. How are the journals in which the articles were published qualified?; 2 - What is the impact of these works in the Web of Science and Google Scholar databases?; 3. What is the impact of these works on the company's network of partnerships?
\end{abstract}

Keywords: Technical-scientific production. Metric analysis Technology transfer. Embrapa Territorial. 


\section{INTRODUÇÃO}

$\mathrm{Na}$ atualidade, um dos desafios impostos às organizações é o de saber transformar, compreender, interpretar e utilizar a enorme massa documental e informacional disponível. Penteado Filho (2009) afirma que a maioria das organizações utiliza entre $10 \%$ e $20 \%$ de toda sua massa informacional, sendo que a informação com potencial de gerar conhecimento não é facilmente encontrada.

Particularmente, nas instituições de pesquisa onde o avanço do conhecimento e a inovação tecnológica são condicionantes para a sua manutenção, a disponibilidade de informações qualificadas é uma medida estratégica que deve ser perseguida pelos gestores de Pesquisa, Desenvolvimento e Inovação (PD\&I). Por conseguinte, à gestão, é imprescindível, que sejam adotadas ações e atividades capazes de identificar aquelas informações que nortearão a geração e desenvolvimento da pesquisa a respeito, por exemplo, de (novas) oportunidades de atuação, adequação dos resultados de pesquisa, prospecção de cenários futuros, etc.

Apoiando essa gestão, os estudos de análise da produção técnico-científica apresentam-se como modelos fundamentais para avaliar o andamento e desenvolvimento de uma determinada área de pesquisa (Santos; Kobashi, 2005). A amplitude de aplicação desses estudos e sua relevância para a gestão de Ciência e Tecnologia (C\&T) permite que sejam utilizados em diferentes abordagens para a estruturação e obtenção de indicadores, tais como o de produtividade científica, que subsidiam a aplicação de recursos, de esforços e, inclusive, a elaboração de políticas públicas de C\&T (Lima et al., 2012).

Como fruto desses estudos, a análise métrica da informação é uma atividade que vem sendo cada vez mais adotada pelas organizações no sentido de apoiá-las em decisões estratégicas (Penteado Filho et al., 2017). Inicialmente aplicados apenas ao contexto científico, passaram a ser utilizados com o objetivo de acompanhar os modelos de comunicação técnico-científica e seus processos de produção, armazenamento, disseminação, recuperação e uso da informação científica e tecnológica registrada com métodos e procedimentos, apropriados à obtenção de indicadores quantitativos relativos aos objetos analisados (Pereira; Fujino, 2015).

A Empresa Brasileira de Pesquisa Agropecuária (EMBRAPA), vinculada ao Ministério da Agricultura, Pecuária e Abastecimento (MAPA), é uma instituição constituída por 43 centros de pesquisas distribuídos pelo país, dentre elas a Embrapa Territorial. Esta 
Unidade foi criada em 2017, após a junção da Embrapa Monitoramento por Satélite, Embrapa Gestão Territorial e o Grupo de Inteligência Territorial Estratégica (GITE), tendo herdado todo o histórico produtivo dessas unidades. Ela atua na geração de conhecimentos, tecnologias e inovações geoespaciais para a agropecuária, por meio da elaboração de zoneamentos, mapeamentos, monitoramentos do uso e da cobertura da terra, além de indicadores de sustentabilidade e competitividade, e outros, nos mais diversos temas. No contexto institucional da Embrapa, as análises da produção técnico-científica são realizadas pela Secretaria de Gestão e Desenvolvimento Institucional (SDI) ${ }^{1}$. Essas análises buscam evidenciar a relevância dos resultados de pesquisa gerados pela empresa e subsidiar o processo de avaliação das Unidades, focando os trabalhos registrados na base de dados Web of Science (WoS).

A gestão da produção técnico-científica da Embrapa é feita pelo sistema Ainfo udesenvolvido e utilizado pelo Sistema Embrapa de Bibliotecas - SEB da Embrapa, que permite o gerenciamento, de forma integrada, de bases de dados documentais e processos bibliográficos, possibilitando o armazenamento, atualização, recuperação e disseminação de informações de forma simples e rápida (Antunes; Oliveira, 1998).

Tendo em vista que muitos dos trabalhos da Embrapa Territorial não se encontram indexados na WoS e que isso pode comprometer a avaliação desta Unidade, faz-se necessário a realização de estudos aprofundados e detalhados sobre o impacto de sua produção científica em outras bases de dados. Assim, o objetivo dessa pesquisa é analisar a relação do impacto da produção técnico científica da Embrapa Territorial nas Bases WoS e Google Scholar (GS). As questões a serem respondidas para o alcance desse objetivo são: 1 - Qual o quantitativo informacional produzido pela Embrapa Territorial? 1.2. Qual a qualificação dos periódicos em que foram registrados os trabalhos no formato de artigos em periódicos; 2 - Qual o impacto dos trabalhos produzidos pela Unidade, nas bases de dados Web of Science e Google Scholar. 3. Qual a relação do impacto dos trabalhos com a rede de parcerias da Unidade.

\footnotetext{
${ }^{1}$ Algumas dessas análises podem ser verificadas em: PENTEADO FILHO, R. de C.; AVILA, A. F. D. Estudo das citações dos artigos da Embrapa na Web of Science de 1977 a 2006. Brasília, DF: Embrapa Informação Tecnológica, 2009. 131 p. (Embrapa-Secretaria de Gestão e Estrategia. Texto para discussão, 37).
} 


\section{CONTEXTO}

$\mathrm{Na}$ Embrapa Territorial, a análise da produção técnico-científica vem sendo empregada pela área de Transferência de Tecnologia (TT) como instrumento auxiliar do processo de gestão da informação técnico-científica, oriunda das ações e atividades de TT e PD\&I praticadas nesta Unidade. A adoção desses estudos visa auxiliar a Unidade na identificação de oportunidades para atuação e abertura de novos mercados, ajustes em suas tecnologias e resultados de pesquisa, novos parceiros, entre outros. Nas análises realizadas, considerando a produção da Embrapa Territorial, percebeu-se que muitos periódicos da área de Geociências, classificados com estrato A1 a B2, não se encontram indexados na WoS, tampouco no Journal Citation Reports (JCR). A WoS é uma das mais conhecidas e respeitadas bases de dados com abordagem métrica. Pertencente ao grupo Thomson Reuters, permite a pesquisa de artigos associados a uma temática e o estabelecimento de conexões entre trabalhos que citam o mesmo trabalho, ou são citados por outros (Falagas et al., 2007). É importante ressaltar que é uma base de acesso restrito que indexa apenas os artigos de revistas indexadas no JCR.

Desta forma e, tendo em vistas estudos na literatura de Ciência da Informação, que estimulam o uso da base de dados Google Scholar (Madruga et al., 2015; Nascimento, 2016; Alves et al., 2017) a área realizou, em 2016, um estudo preliminar a respeito do impacto dos trabalhos desta Unidade, considerando a base GS (Pinto et al., 2016). De acordo com Nascimento (2016), a base oferece uma série de recursos para análise de artigos e periódicos reunindo conteúdos de diferentes fontes. E inclui diversos formatos de publicação, não se restringindo apenas ao artigo em periódico.

\section{MATERIAL E MÉTODOS}

Trata-se de uma pesquisa quali-quantitativa, de natureza exploratória, que busca investigar o desempenho dos artigos publicados pela Embrapa Territorial, no período de 2010 a $2015^{2}$, a partir da variável "número de citações", com vistas a reconhecer o impacto gerado pelas pesquisas realizadas nesta Unidade da Embrapa, na comunidade técnicocientífica. Para condução deste trabalho foram estruturadas 3 etapas:

\footnotetext{
2 O período estudado representa a investidura desta Unidade da Embrapa em produzir uma carteira de projetos de pesquisa com novas frentes de atuação.
} 


\section{Etapa 1: Identificação e extração do corpus de estudo.}

Nesta etapa foram extraídos os dados referentes à produção técnico-científica da Embrapa Territorial, no período de 2010 a 2018, registrados no repositório Ainfo $^{3}$ e sua qualificação por meio da organização dos artigos, por tipo de classificação Qualis Capes. Foram realizados os seguintes procedimentos:

- levantamento de indicadores capazes de subsidiar as respostas para as questões apresentadas.

- a extração dos dados referentes à produção técnico-científica da Unidade presentes na base Ainfo,

- $\quad$ para a identificação da classificação dos artigos de periódicos foi utilizada a lista com as classificações da Embrapa, que segue a Qualis Capes. Quando algum título de periódico não estava na relação da Embrapa foi realizada consulta na base Sucupira, para levantamento desta informação.

- complementarmente à classificação Qualis/Embrapa do periódico foram identificados o Fator de impacto no JCR e o Scimago Journal Ranking, para refinar a qualidade do periódico, mas este trabalho não se propôs a discutir a relação entre esses índices. Essa identificação teve o objetivo principal de registrar a posição de um periódico em nos três índices.

\section{Etapa 2: Identificação do impacto dos trabalhos componentes do "corpus de estudo", a partir da variável "citação".}

Nesta etapa, buscou-se comparar o impacto dos artigos de periódicos nas bases de dados: Google Scholar (GS) e Web of Science (WoS). Para isto, foram adotados os seguintes procedimentos:

- Cruzamento automático de dados desta investigação, no software Publish or Perish. Foi usado como parâmetro o título do artigo, visando levantar o número de citações recebidas, por cada artigo publicado, na base Google Scholar;

- Investigação na base $W o S$, para identificação dos trabalhos levantados na etapa 1, que possuíam registro nesta base, utilizando como meio para recuperação desses

\footnotetext{
${ }^{3}$ Ressalta-se que tal repositório é gerido pela equipe de profissionais da informação da Embrapa, para a gestão da informação técnico-científica produzida pela empresa.
} 
trabalhos:

- Delimitação das expressões a serem utilizadas para recuperação da informação na WoS, onde foram inseridas as denominações "Embrapa Terri*”; Embrapa Sat*; Centro de Pesquisa do Monit*

- Identificação dos artigos de periódicos recuperados na WoS, para levantamento do número de citação, utilizando como o ambiente de "análise" da WoS.

\section{Etapa 3: Exploração visual dos resultados}

Esta etapa teve como principal objetivo produzir análises nos softwares específicos: as tabelas dinâmicas do Excel, os gráficos interativos do Google Drive e a ferramenta Tableau Public, para cruzamento e visualização de indicadores, capazes de responder às três questões apresentadas neste trabalho.

\section{RESULTADOS E DISCUSSÃO}

Os resultados serão apresentados no formato de respostas às questões apresentadas na introdução deste trabalho. Neste aspecto, foram levantados seis indicadores para subsidiar a realização da pesquisa:

1. Produção técnico-científica da Unidade: evidencia o quantitativo de trabalhos, por tipologia documental.

2. Qualificação dos artigos de periódicos: mostra o estrato Qualis Capes dos trabalhos produzidos pela Unidade.

3. Impacto da Produção: apresenta a repercussão, na comunidade técnicocientífica, dos trabalhos gerados pela Unidade por meio da variável 'citações';

4. Principais periódicos onde os trabalhos foram publicados: verifica a ocorrência de repetições de artigos por periódicos;

5. Número de parceiros: identifica o quantitativo de parceiros envolvidos nos trabalhos da Unidade;

6. Rede de colaboradores: identifica os parceiros e suas relações com os trabalhos mais relevantes da Unidade, por meio do impacto obtido pela variável 'citação'.

Questão 1 - Qual o quantitativo informacional produzido pela Embrapa Territorial? 1.1. Qual 
a qualificação dos periódicos em que foram registrados os trabalhos no formato de artigos em periódicos;

Indicador 1: Produção técnico-científica da Unidade

No período analisado, a Embrapa Territorial publicou um total de 859 trabalhos, em 16 tipologias documentais distintas, correspondendo a 142 itens/ano. As comunicações em eventos, representadas pelas tipologias "Artigo em anais de congresso" e "Resumo em anais de congresso", representaram $47 \%$ deste total. Desta forma, pode-se inferir que quase a metade dos trabalhos publicados buscam a comunicação em eventos, onde é possível identificar parcerias e ajustes necessários para o trabalho apresentado.

Tabela 1 - Produção científica da Embrapa Monitoramento por Satélite no período de 2010 a 2015 .

\section{Tipo de Publicação ${ }^{4}$}

Artigo em Periódico Indexado

Artigo em anais de congresso

Resumo em anais de congresso

Organização/Edição de Livros

Capítulo em Livro Técnico-Científico

Circular Técnica/Comunicado Técnico

Série Documentos

Boletim de Pesquisa e Desenvolvimento

Folder/Folheto/Cartilha

Orientação de Tese de Pós-Graduação

Artigo de Divulgação na Mídia

Monitoramento/Zoneamento

Software

Nota Técnica

Programa de Rádio

Vídeo/DVD

\begin{tabular}{|c|c|c|c|c|c|c|}
\hline & 11 & 22 & 24 & 27 & 35 & 33 \\
\hline & 34 & 59 & 39 & 48 & 41 & 69 \\
\hline & 17 & 34 & 20 & 10 & 13 & 21 \\
\hline & 2 & 0 & 0 & 3 & 1 & 1 \\
\hline fico & 11 & 11 & 6 & 8 & 27 & 14 \\
\hline nico & 2 & 1 & 14 & 8 & 1 & 2 \\
\hline & 7 & 8 & 8 & 8 & 2 & 4 \\
\hline mento & 8 & 9 & 2 & 0 & 1 & 2 \\
\hline & 1 & 14 & 4 & 2 & 3 & 7 \\
\hline ก̃̃ & 4 & 1 & 0 & 2 & 0 & 0 \\
\hline & 13 & 10 & 13 & 11 & 10 & 3 \\
\hline & 1 & 0 & 0 & 4 & 9 & 3 \\
\hline & 1 & 0 & 1 & 3 & 1 & 3 \\
\hline & 0 & 0 & 0 & 0 & 0 & 0 \\
\hline & 0 & 1 & 1 & 2 & 1 & 0 \\
\hline & 0 & 0 & 0 & 1 & 1 & 0 \\
\hline TOTAL (ANO) & 112 & 170 & 132 & 137 & 146 & 162 \\
\hline TOTAL (GERAL) & 859 & & & & & \\
\hline
\end{tabular}

Fonte: Ainfo (2017).

\footnotetext{
${ }^{4}$ Tipologias disponíveis no Ainfo.
} 
Indicador 2. Qualificação dos artigos de periódicos

Ao interpretar a tipologia "Artigo em Periódico Indexado" verificou-se que a evolução qualitativa para essa produção esteve concentrada nos anos de 2014 e 2015, período em que vários projetos e ações foram finalizados na Unidade, apresentando, assim, seus resultados.

Para qualificar a produção técnico-científica de seus pesquisadores, a Embrapa adota a classificação Qualis Capes, usando sempre o maior estrato do periódico, independente da área do conhecimento que está sendo avaliada. A Qualis Capes é uma classificação que emprega 8 tipos de estratos indicativos da qualidade, para classificar os periódicos nacionais A1, o mais elevado; A2; B1; B2; B3; B4; B5; C, tendo este último peso zero.

No total, a Unidade produziu 152 artigos científicos, representando uma média de 25,5 artigos/ano. Analisando a partir dos extratos da Qualis Capes, observou-se que 110 dos artigos publicados estão classificados nos níveis A1 a B2, considerados os mais desejados e representa a faixa mais alta para a avaliação das Unidades da Embrapa. Este conjunto representa um percentual de $72 \%$ dos artigos publicados, conforme a seguir:

Tabela 2 - Quantidade de artigos em periódicos produzidos pela Unidade, segundo classificação da Capes.

\begin{tabular}{|c|cccccccccccc}
\hline Ano & A1 & A2 & B1 & B2 & B3 & B4 & B5 & C & TOTAL (Ainfo) & TOTAL( WoS) & TOTAL( GS) \\
\hline 2010 & 1 & 0 & 6 & 0 & 2 & 1 & 1 & 0 & 11 & 1 & 9 \\
2011 & 5 & 3 & 8 & 1 & 1 & 3 & 0 & 1 & 22 & 9 & 18 \\
2012 & 2 & 13 & 1 & 2 & 1 & 3 & 0 & 2 & 24 & 13 & 24 \\
2013 & 6 & 6 & 2 & 4 & 3 & 4 & 1 & 1 & 27 & 5 & 25 \\
2014 & 8 & 4 & 9 & 4 & 4 & 3 & 2 & 1 & 35 & 9 & 33 \\
2015 & 5 & 7 & 8 & 5 & 0 & 2 & 2 & 3 & 32 & 8 & 27 \\
\hline TOTAL GERAL & & & & & & & & & $\mathbf{1 5 2}$ & $\mathbf{4 5}$ & $\mathbf{1 3 6}$ \\
\hline
\end{tabular}

Nesta tabela $2^{5}$ também é possível verificar, por ano, quantos artigos do total (152) encontram-se indexados na Web of Science e Google Scholar. Enquanto na primeira encontram-se registrados 45 , ou $29 \%$, do total de artigos produzidos pela Embrapa Territorial, a segunda alcançou o número de 136, ou 89,4\%, artigos indexados.

\footnotetext{
${ }^{5} \mathrm{O}$ conteúdo da tabela 2 pode ser visualizado no formato de gráfico, disponível em: 〈 bit.ly/cnpm artigos $>$.
} 


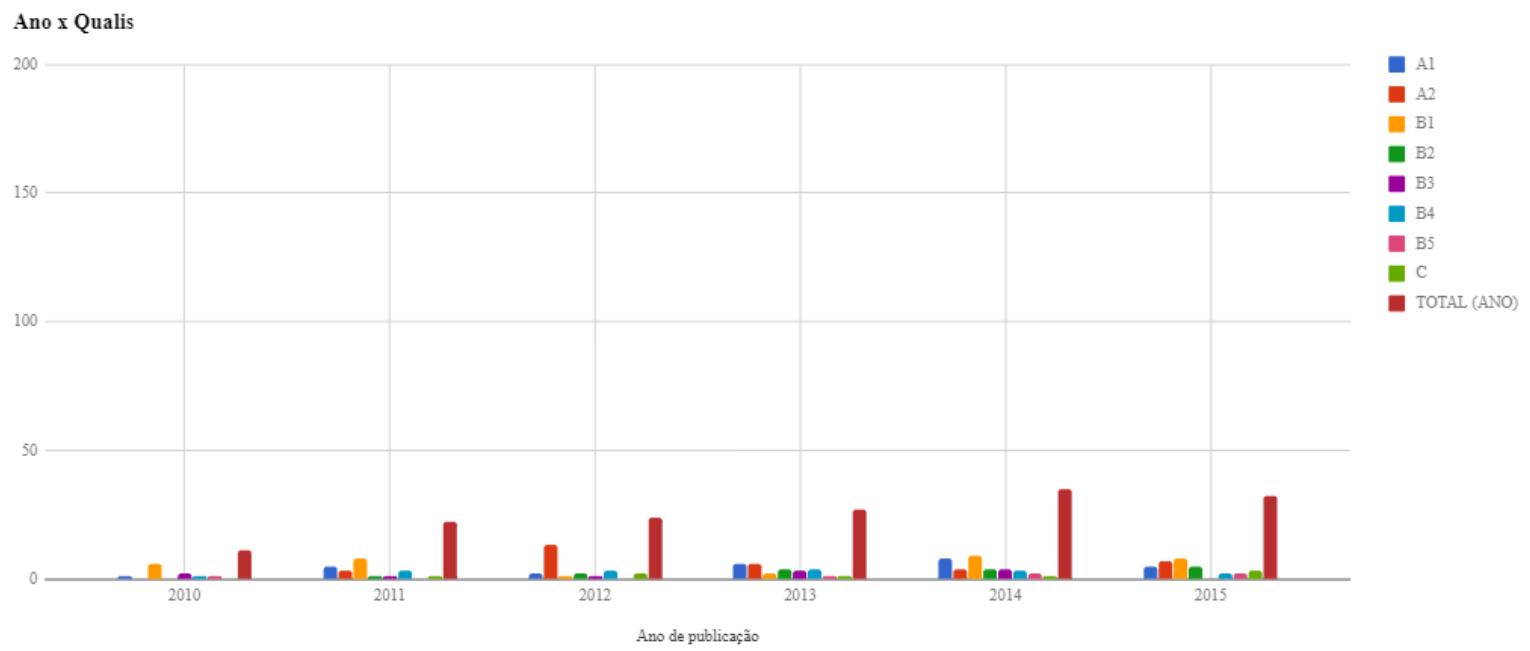

Questão 2 - Qual o impacto dos trabalhos produzidos pela Unidade, nas bases de dados Web of Science e Google Scholar.

Indicador 3. Impacto da Produção ${ }^{6}$

A partir do quantitativo de artigos publicados, verificou-se o impacto dos trabalhos nas duas bases de dado. Foi possível observar que a base Google Scholar apresenta o maior número de citações/impacto, conforme tabela 3. A coluna "Total de citações" da tabela 3 reúne as informações sobre o impacto total de citações, do conjunto de artigos recuperados em cada base de dados. O impacto da produção, medido pelo quantitativo de citações foi realizado comparativamente nas duas bases: Web of Science e Google Scholar.

Neste aspecto:

$\rightarrow$ pode-se verificar que o número de artigos indexados na WoS representa menos de $30 \%$ do total produzido pela Unidade e possui uma média de citações igual a 10,4/artigo, conforme Tabela 3.

$\rightarrow$ na base Google Scholar, o volume de trabalhos recuperados com uso do software Publish or Perish para verificação do impacto é maior, mas a média de citações por artigo é inferior à identificada na $W o S$, representando 9 citações por artigo.

\footnotetext{
${ }^{6}$ Levantamento para verificação de impacto realizado até 31 de março de 2016, nas bases WoS e Google Scholar .
} 
Tabela 3 - Impacto dos artigos da Unidade nas bases WoS e GS

\begin{tabular}{|c|c|c|c|}
\hline Base & Qtd Artigos Indexados & Total de citações & Média de citações por artigo \\
\hline WoS & 45 & 469 & 10,4 \\
\hline Scholar ${ }^{7}$ & 136 & 1246 & 9 \\
\hline
\end{tabular}

$\mathrm{Na}$ comparação entre as bases estudadas, foram selecionados os cinco primeiros trabalhos mais citados na Web of Science e no Google Scholar. Nesta análise, notou-se uma relação de recorrência para três títulos, havendo uma variação no ranking desses três para o título "Classifying multiyear agricultural land use data from Mato Grosso using time-series MODIS vegetation index data", que ocupa o terceiro lugar na WoS e quarto lugar na Google Scholar (Tabela 4).

Tabela 4 - Comparação entre os 5 primeiros trabalhos mais citados na base Web of Science em relação ao Google Scholar.

\begin{tabular}{|c|l|c|c|c|c|}
\hline \multirow{2}{*}{ Item } & \multicolumn{1}{|c|}{ Título } & $\begin{array}{c}\text { Posição } \\
\text { na WoS }\end{array}$ & $\begin{array}{c}\text { Posição } \\
\text { no GS }\end{array}$ & $\begin{array}{c}\text { Citação } \\
\text { WoS }\end{array}$ & $\begin{array}{c}\text { Citação } \\
\text { GS }\end{array}$ \\
\hline 1 & $\begin{array}{l}\text { The Amazon basin in transition. Nature, v. } \\
\text { 481, p. 321-328, Jan. 2012. }\end{array}$ & 1 & 1 & 186 & 295 \\
\hline 3 & $\begin{array}{l}\text { Framing sustainability in a telecoupled } \\
\text { world. Ecology and Society, v. 18, n. 2, jun. } \\
\text { 2013. 19 p. }\end{array}$ & 2 & 2 & 64 & 130 \\
\hline $\begin{array}{l}\text { Classifying multiyear agricultural land use } \\
\text { data from Mato Grosso using time-series } \\
\text { MODIS vegetation index data. Remote } \\
\text { Sensing of Environment, New York, v. 130, } \\
\text { p. 39-50, 2013. }\end{array}$ & 3 & 4 & 32 & 49 \\
\hline 4 & $\begin{array}{l}\text { A comparative analysis of ALOS PALSAR } \\
\text { L-band and RADARSAT-2 C-band data for } \\
\text { land-cover classification in a tropical moist }\end{array}$ & 4 & 8 & 24 & 35 \\
\hline
\end{tabular}

\footnotetext{
7 Verificação das citações recebidas artigo a artigo, a partir de levantamento no Ainfo, por meio do software Publish or Perish. Encontrados 139 trabalhos, dos 152.
} 


\begin{tabular}{|c|c|c|c|c|c|}
\hline & $\begin{array}{l}\text { region. ISPRS Journal of Photogrammetry } \\
\text { and Remote Sensing, v. } 70 \text {, p. } 26-38,2012 . \\
\text { p. } 26-38 .\end{array}$ & & & & \\
\hline 5 & $\begin{array}{l}\text { A social and ecological assessment of } \\
\text { tropical land uses at multiple scales: the } \\
\text { sustainable Amazon network. Philosophical } \\
\text { Transactions of the Royal Society } \\
\text { Biological Sciences, v. } 368, \text { n. } 1619, \text { p. } 1 \text { - } \\
11,2013 \text {. }\end{array}$ & 5 & 6 & 21 & 39 \\
\hline 6 & $\begin{array}{l}\text { Aboveground forest biomass estimation } \\
\text { with Landsat and LiDAR data and } \\
\text { uncertainty analysis of the estimates. } \\
\text { International Journal of Forestry Research, } \\
\text { v. 2012. p. 16, } 201216 \text { p. }\end{array}$ & $\begin{array}{c}\text { Não } \\
\text { indexado }\end{array}$ & 3 & $\begin{array}{c}\text { Não } \\
\text { indexado }\end{array}$ & 57 \\
\hline 7 & $\begin{array}{l}\text { Forest Cover Changes in Tropical South and } \\
\text { Central America from } 1990 \text { to } 2005 \text { and } \\
\text { Related Carbon Emissions and Removals. } \\
\text { Remote Sensing, v. 4, p. } 1369-1391,2012 . \\
\text { p. } 1369-1391 .\end{array}$ & $\begin{array}{c}\text { Não } \\
\text { indexado }\end{array}$ & 5 & - & 41 \\
\hline TOTAL & & & & 327 & 646 \\
\hline
\end{tabular}

Notou-se, ainda, que no GS, os trabalhos possuem mais citações do que os mesmos títulos na WoS. Isso permite inferir que o GS inclui um número maior de bases indexadas, enquanto que a WoS é mais restritiva. Além do que, as bases possuem formas diferenciadas de acesso. Enquanto o GS está disponível gratuitamente, a WoS não.

Indicador 4. Principais Periódicos onde os trabalhos foram publicados ${ }^{8}$

Em relação aos periódicos, os 152 trabalhos foram publicados em 85 títulos de periódicos e $10 \%$ destes títulos tiveram mais do que cinco artigos publicados pela Unidade. Buscando entender essa ocorrência, identificou-se como prováveis motivos: 1. dificuldade em publicar em periódicos da área de Geociências, 2. a pouca disponibilidade de títulos de periódicos da área Geociências e 3. a falta de domínio em língua estrangeira.

\footnotetext{
${ }^{8}$ Dados do sistema Ainfo.
} 
Analisando o número de artigos por periódicos e as citações recebidas na base Google Scholar, percebeu-se que o impacto no número de citações está diretamente relacionado à qualidade do periódico, conforme Tabela 5.

Tabela 5 - Título do periódico e classificações pelo número de citações no Google Scholar

\begin{tabular}{|c|c|c|c|c|c|}
\hline Periódico & Qtd. & Qualis/Embrapa & $\begin{array}{l}\text { Fator de } \\
\text { impacto }\end{array}$ & SJR & $\begin{array}{c}\text { Citações } \\
\text { G. } \\
\text { Scholar }\end{array}$ \\
\hline Agroanalysis & 10 & B3 & Não possui & $\begin{array}{l}\text { Não } \\
\text { possui }\end{array}$ & 4 \\
\hline Bragantia & 9 & B1 & 0,62 & Q1 & 76 \\
\hline Engenharia na Agricultura & 6 & B2 & Não possui & Q1 & 11 \\
\hline Geografia & 9 & A2 & Não possui & $\begin{array}{l}\text { Não } \\
\text { possui }\end{array}$ & 28 \\
\hline $\begin{array}{l}\text { International Journal of Remote } \\
\text { Sensing }\end{array}$ & 6 & $\mathrm{~A} 1$ & 1.359 & Q1 & 133 \\
\hline MundoGEO & 8 & B4 & Não possui & $\begin{array}{l}\text { Não } \\
\text { possui }\end{array}$ & 4 \\
\hline Pesquisa Agropecuária Brasileira & 12 & A1 & 0,676 & Q1 & 98 \\
\hline Proceedings of SPIE & 7 & B2 & Não possui & Q1 & 5 \\
\hline
\end{tabular}

A qualificação de periódicos, na tabela 5, é representada pelas colunas Qualis/Embrapa; Fator de impacto JCR e SJR. Neste sentido, nota-se que quanto maior a classificação, maior o número de citações/impacto/relevância dos artigos. Essa é uma constatação comum aos estudos bibliométricos que tentam compreender a relação entre qualidade e impacto Sandes-Guimarães (2016).

Questão 3 - 2 - Qual a relação do impacto dos trabalhos com as parcerias da Unidade.

Indicador 5. Número de parceiros ${ }^{9}$

A rede de colaboradores, ou autores da Unidade totalizou 1.180 indivíduos, representando uma média de 6,11 autores por artigo publicado. Desse total, 340 indivíduos são empregados de outras Unidades da Embrapa e 820 de instituições parceiras, os quais estão ligados a mais de duas centenas de instituições nacionais e internacionais, conforme pode ser visto no próximo Indicador 6. Rede de colaboradores.

\footnotetext{
${ }^{9}$ Considerando, também, os autores da Unidade.
} 
Indicador 6. Rede de Colaboradores

No período de 2010 a 2015, a Unidade estabeleceu 202 parcerias com instituições de ensino superior, pesquisa, órgãos públicos e privados, nacionais e internacionais. As instituições brasileiras ocuparam o papel de destaque nessas parcerias, representando $52 \%$ do total do número de parceiros. O Sudeste foi a região brasileira com maior número de instituições, seguido da região Norte e Nordeste, conforme Gráfico 2. Parcerias por regiões do Brasil.

Gráfico 2 - Parcerias por regiões do Brasil

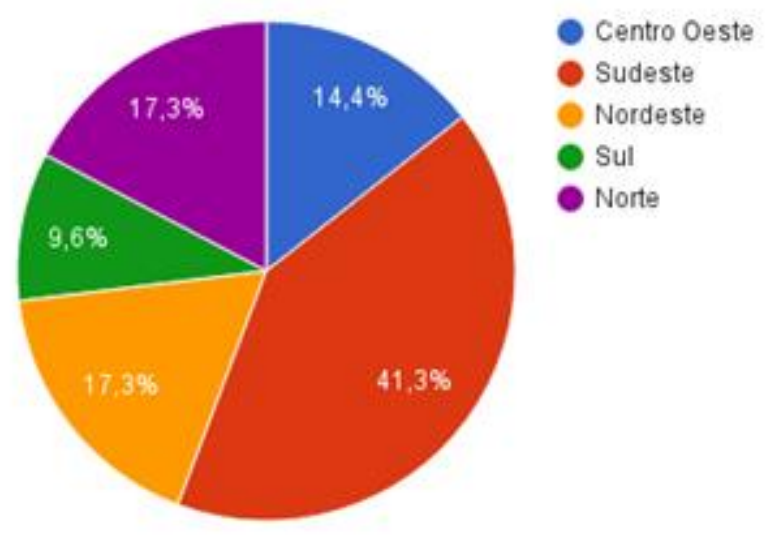

Ao todo a Unidade estabeleceu parceria com 96 instituições internacionais, situadas em 27 países, localizados em 5 continentes: América do Norte, América do Sul, Ásia, Austrália, Europa, destacando-se a Rússia, país de maior extensão territorial do mundo (Europa e Ásia) conforme a Tabela 6. 
Tabela 6 - Relação das parcerias estabelecidas pela Unidade no período de 2010 a $2015^{10}$

\begin{tabular}{|c|c|c|c|}
\hline Parceiros & País & Município & $\begin{array}{l}\text { Região / } \\
\text { Continente }\end{array}$ \\
\hline ANA & Brazil & Brasília & Centro Oeste \\
\hline ANHANGUERA EDUCACIONAL & Brazil & Campinas & Sudeste \\
\hline APPLIED GEOSOLUTIONS & USA & New Hampshire & EUA \\
\hline APTA & Brazil & São Paulo & Sudeste \\
\hline ASSOCIAÇÃO PLANTAS DO NORDESTE & Brazil & Recife & Nordeste \\
\hline AGRICULTURE \& AGRI-FOOD CANADA & Canadá & Ontário & Canadá \\
\hline $\begin{array}{l}\text { ALPEN ADRIA UNIVERSITY KLAGENFURT- } \\
\text { WIEN-GRAZ }\end{array}$ & Áustria & Klagenfurt & Europa \\
\hline $\begin{array}{l}\text { AMERICAN ASSOCIATION FOR THE } \\
\text { ADVANCEMENT }\end{array}$ & USA & Washington & EUA \\
\hline ASSOCIAÇÃO DE PLANTAS DO NORDESTE & Brazil & Recife & Nordeste \\
\hline BOSTON UNIVERSITY & USA & Boston & EUA \\
\hline CALIFORNIA INSTITUTE OF TECHNOLOGY & USA & California & EUA \\
\hline CECAT & Brazil & Brasília & Centro Oeste \\
\hline CELULOSE NIPO-BRASILEIRA & Brazil & Belo Horizonte & Sudeste \\
\hline CENA/USP & Brazil & Piracicaba & Sudeste \\
\hline CHINA AGRICULTURAL UNIVERSITY & China & Pequim & Ásia \\
\hline CHINESE ACADEMY OF SCIENCES & China & Pequim & Ásia \\
\hline CIRAD & France & Paris & Europa \\
\hline CNPA (EMBRAPA) & Brazil & Seropédica & Sudeste \\
\hline CNPAB (EMBRAPA) & Brazil & Seropédica & Sudeste \\
\hline CNPDIA (EMBRAPA) & Brazil & São Carlos & Sudeste \\
\hline CNPF (EMBRAPA) & Brazil & Colombo & Sul \\
\hline CNPGL (EMBRAPA) & Brazil & Juiz de Fora & Sudeste \\
\hline $\begin{array}{l}\text { USDA/INTERNATIONAL INSTITUTE OF } \\
\text { TROPICAL FORESTRY }\end{array}$ & USA & Washington & EUA \\
\hline CNPMF (EMBRAPA) & Brazil & Cruz das Almas & Nordeste \\
\hline CNPMS (EMBRAPA) & Brazil & Sete Lagoas & Sudeste \\
\hline CNPS (EMBRAPA) & Brazil & Rio de Janeiro & Sudeste \\
\hline CNPTIA (EMBRAPA) & Brazil & Campinas & Sudeste \\
\hline CNPUV (EMBRAPA) & Brazil & Bento Gonçalves & Sul \\
\hline CNPQ & Brazil & Brasília & Centro Oeste \\
\hline COLUMBIA UNIVERSITY & USA & New York & EUA \\
\hline COROLAB HUMBOLDT & Venezuela & Caracas & América do $\mathrm{Su}$ \\
\hline CPAC (EMBRAPA) & Brazil & Planaltina & Centro Oeste \\
\hline CPAF-AC (EMBRAPA) & Brazil & Rio Branco & Norte \\
\hline CPAF-RR (EMBRAPA) & Brazil & Roraima & Norte \\
\hline
\end{tabular}

10 Pode ser visualizada em $\langle\underline{\text { https://goo.gl/1fWjMU }}>$. 


\begin{tabular}{|c|c|c|c|}
\hline CPAP (EMBRAPA) & Brazil & Corumbá & Centro Oeste \\
\hline CPATC (EMBRAPA) & Brazil & Pelotas & Sul \\
\hline CPATSA (EMBRAPA) & Brazil & Petrolina & Nordeste \\
\hline CPATU (EMBRAPA) & Brazil & Belém & Norte \\
\hline CPPSE (EMBRAPA) & Brazil & São Carlos & Sudeste \\
\hline CRANFIELD UNIVERSITY & England & Cranfield & Europa \\
\hline CALIFORNIA INSTITUTE OF TECHNOLOGY & USA & California & EUA \\
\hline CARNEGIE INSTITUTION FOR SCIENCE & USA & Washington & EUA \\
\hline COLORADO STATE UNIVERSITY & USA & Fort Collins & EUA \\
\hline DATA+ MOSCOW & Rússia & Moscou & Rússia \\
\hline DENVER BOTANIC GARDENS & USA & Denver & EUA \\
\hline DOLAN INTEGRATION GROUP & USA & Westminster & EUA \\
\hline DPD & Brazil & Brasília & Centro Oeste \\
\hline ESALQ/USP & Brazil & Piracicaba & Sudeste \\
\hline $\begin{array}{l}\text { EASTERN CEREAL \& OIL SEED RESEARCH } \\
\text { CENTRE }\end{array}$ & Canadá & Ontário & Canadá \\
\hline FFCLRP & Brazil & Além Paraíba & Sudeste \\
\hline FLORIDA GULF COAST UNIVERSITY & USA & Flórida & EUA \\
\hline FRIEDRICH SCHILLER UNIVERSITY & Germany & Turíngia & Europa \\
\hline $\begin{array}{l}\text { FUNDAÇÃO MUNICIPAL DO MEIO AMBIENTE } \\
\text { DE ITAJAÍ }\end{array}$ & Brazil & Itajaí & Sul \\
\hline FOREST SECTOR INSIGHTS AB & Sweden & Hedemora & Europa \\
\hline HERBÁRIO EAFM & Brazil & Manaus & Norte \\
\hline IAC & Brazil & Campinas & Sudeste \\
\hline IMAZON & Brazil & Belém & Norte \\
\hline INPA & Brazil & Manaus & Norte \\
\hline INPE & Brazil & $\begin{array}{l}\text { São José dos } \\
\text { Campos }\end{array}$ & Sudeste \\
\hline INSTITUTE FOR ENVIRONMENTAL STUDIES & Netherlands & Amsterdan & Europa \\
\hline $\begin{array}{l}\text { INSTITUTE FOR GLOBAL ENVIRONMENTAL } \\
\text { STRATEGIES }\end{array}$ & Japan & Kanagawa & Ásia \\
\hline INSTITUTO AGRONÔMICO & Brazil & Campinas & Sudeste \\
\hline INSTITUTO DE BOTÂNICA & Brazil & São Paulo & Sudeste \\
\hline INSTITUTO DE ZOOTECNIA NOVA ODESSA & Brazil & Nova Odessa, SP & Sudeste \\
\hline INSTITUTO NACIONAL DO SEMIÁRIDO & Brazil & $\begin{array}{l}\text { Campina Grande, } \\
\text { PB }\end{array}$ & Sudeste \\
\hline INSTITUTO DE CLIMA Y ÁGUA-BUENOS AIRES & Argentina & Buenos Aires & América do Sul \\
\hline $\begin{array}{l}\text { INTERNATIONAL UNION FOR CONSERVATION } \\
\text { OF NATURE }\end{array}$ & Switzerland & Gland & Europa \\
\hline IPEA & Brazil & Rio de Janeiro & Sudeste \\
\hline ISPRA & Italy & Varese & Europa \\
\hline ITA & Brazil & São José dos & \\
\hline
\end{tabular}




\begin{tabular}{|c|c|c|c|}
\hline INDIANA UNIVERSITY & USA & Bloomington & EUA \\
\hline $\begin{array}{l}\text { INTERNATIONAL INSTITUTE FOR APPLIED } \\
\text { SYSTEMS ANALYSIS }\end{array}$ & Áustria & Luxemburgo & Europa \\
\hline JET PROPULSION LABORATORY & USA & Pasadena, CA & EUA \\
\hline JRC & Italy & Ispra, VA & Europa \\
\hline JARDIN BOTANICO DE MISSOURI & USA & Saint Louis, MO & EUA \\
\hline KANSAS APPLIED REMOTE SENSING & USA & Lawrence, KA & EUA \\
\hline KASETSART UNIVERSITY & Thailand & Bangkok & Ásia \\
\hline $\begin{array}{l}\text { KING MONGKUT'S UNIVERSITY OF } \\
\text { TECHNOLOGY }\end{array}$ & Thailand & Bangkok & Ásia \\
\hline LANCASTER UNIVERSITY & England & Lancaster & Europa \\
\hline $\begin{array}{l}\text { LEIBNIZ INSTITUTE OF FRESHWATER } \\
\text { ECOLOGY AND INLAND FISHERIES }\end{array}$ & Germany & Stechlin & Europa \\
\hline LONDON SCHOOL OF ECONOMICS & England & Londres & Europa \\
\hline LUND UNIVERSITY & Sweden & Lund & Europa \\
\hline MAEC-AECID/ESPANHA & Espanha & Madri & Europa \\
\hline $\begin{array}{l}\text { MASSACHUSETTS INSTITUTE OF } \\
\text { TECHNOLOGY }\end{array}$ & USA & Cambridge, MA & EUA \\
\hline MICHIGAN STATE UNIVERSITY & USA & East Lansing, MI & \\
\hline MINISTÉRIO DA SAÚDE & Brazil & Brasília, DF & \\
\hline MINISTÉRIO DE CIÊNCIA E TECNOLOGIA & Brazil & Brasília & Centro Oest \\
\hline MONASH UNIVERSITY & Austrália & Clayton & Austrália \\
\hline MUSEU EMÍLIO GOELDI & Brazil & Belém & Norte \\
\hline $\begin{array}{l}\text { MUSEUM NATIONAL D'HISTOIRE NATURELLE } \\
\text { - FRANCE }\end{array}$ & France & Paris & Europa \\
\hline NASA & USA & Washington & EUA \\
\hline $\begin{array}{l}\text { NATIONAL INSTITUTE FOR ENVIRONMENTAL } \\
\text { STUDIES }\end{array}$ & Japan & Tsukuba & Ásia \\
\hline $\begin{array}{l}\text { NATIONAL RESEARCH INSTITUTE OF SCIENCE } \\
\text { AND TECHNOLOGY FOR ENVIRONMENT AND } \\
\text { AGICULTURE }\end{array}$ & France & Paris & Europa \\
\hline NEPTUNE AND COMPANY & USA & Lakewood, CO, & EUA \\
\hline OREGON STATE UNIVERSITY & USA & Corvallis, OR & EUA \\
\hline USDA & USA & Washington, DC & EUA \\
\hline PUC-RJ & Brazil & Rio de Janeiro, RJ & Sudeste \\
\hline PURDUE UNIVERSITY & USA & $\begin{array}{l}\text { West Lafayette, } \\
\text { IN }\end{array}$ & EUA \\
\hline PUCCAMP & Brazil & Campinas, SP & Sudeste \\
\hline ROYAL BOTANIC GARDENS & England & Surrey & Europa \\
\hline SAN JUAN FOREST SERVICE & USA & Bayfield, CO & EUA \\
\hline $\begin{array}{l}\text { SCHOOL OF MATHEMATICAL AND } \\
\text { GEOSPATIAL SCIENCES }\end{array}$ & Australia & Melbourne & Australia \\
\hline
\end{tabular}




\begin{tabular}{|c|c|c|c|}
\hline SEDE & Brazil & Brasília, DF & Centro Oeste \\
\hline SGE & Brazil & Brasília, DF & Centro Oeste \\
\hline SGTE & Brazil & Campinas, SP & Sudeste \\
\hline SIPAM & Brazil & Manaus, AM & Norte \\
\hline $\begin{array}{l}\text { SMITHSONIAN ENVIRONMENTAL RESEARCH } \\
\text { CENTER }\end{array}$ & USA & Edgewater, MD & EUA \\
\hline $\begin{array}{l}\text { SMITHSONIAN TROPICAL RESEARCH } \\
\text { INSTITUTE }\end{array}$ & Panamá & Ancon & \\
\hline $\begin{array}{l}\text { SOUTHERN ILLINOIS UNIVERSITY AT } \\
\text { CARBONDALE }\end{array}$ & USA & Carbondale, IL & EUA \\
\hline SPATIAL INFORMATICS GROUP & USA & Pleasanton, CA & EUA \\
\hline STANFORD UNIVERSITY & USA & Stanford, CA & EUA \\
\hline SCHOOL OF GEOGRAPHY & England & Leeds & Europa \\
\hline $\begin{array}{l}\text { SECRETARIA MUNICIPAL DE COMÉRCIO, } \\
\text { INDÚSTRIA, SERVIÇOS E TURISMO DE } \\
\text { CAMPINAS }\end{array}$ & Brazil & Campinas, SP & Sudeste \\
\hline STATE UNIVERSITY OF NEW YORK & USA & New York & EUA \\
\hline STOCKHOLM UNIVERSITY & Sweden & Stockholm & Europa \\
\hline THE NATURE CONSERVANCY & USA & Virgínia & EUA \\
\hline THE WOODS HOLE RESEARCH CENTER & USA & Falmouth, MA & EUA \\
\hline $\begin{array}{l}\text { U.S. ENVIRONMENTAL PROTECTION AGENCY } \\
\text { OFFICE OF RESEARCH AND DEVELOPMENT }\end{array}$ & USA & Corvallis, OR & EUA \\
\hline UEFS & Brazil & $\begin{array}{l}\text { Feira de Santana, } \\
\text { BA }\end{array}$ & Sudeste \\
\hline UENF & Brazil & $\begin{array}{l}\text { Campos dos } \\
\text { Goytacazes, RJ }\end{array}$ & Sudeste \\
\hline UEPB & Brazil & $\begin{array}{l}\text { Campina Grande, } \\
\text { PB }\end{array}$ & Nordeste \\
\hline UERJ & Brazil & Rio de Janeiro, RJ & Sudeste \\
\hline UESC & Brazil & Ilhéus, BA & Nordeste \\
\hline UFAC & Brazil & Rio Branco, AC & Norte \\
\hline UFAM & Brazil & Manaus, AM & Norte \\
\hline UFBA & Brazil & Salvador, BA & Nordeste \\
\hline UFCG & Brazil & $\begin{array}{l}\text { Campina Grande, } \\
\text { PB }\end{array}$ & Nordeste \\
\hline UFMG & Brazil & $\begin{array}{l}\text { Belo Horizonte, } \\
\text { MG }\end{array}$ & Sudeste \\
\hline UFMT & Brazil & Cuiabá, MT & Centro Oeste \\
\hline UFOPA & Brazil & Santarém, PA & Norte \\
\hline UFPE & Brazil & Recife, PE & Nordeste \\
\hline UFPI & Brazil & Teresina, PI & Nordeste \\
\hline UFPR & Brazil & Curitiba, PR & Sul \\
\hline UFRO & Brazil & Porto Velho & Norte \\
\hline
\end{tabular}




\begin{tabular}{|c|c|c|c|}
\hline UFRJ & Brazil & Rio de Janeiro & Sudeste \\
\hline IFRO & Brazil & Porto Velho, RO & Norte \\
\hline UFRPE & Brazil & Recife, PE & Nordeste \\
\hline UFRRJ & Brazil & Seropédica, RJ & Sudeste \\
\hline UFSC & Brazil & Florianópolis, SC & Sul \\
\hline UFSCAR & Brazil & São Carlos, SP & Sudeste \\
\hline UFSM & Brazil & Santa Maria, RS & Sul \\
\hline UFT & Brazil & Palmas, TO & Norte \\
\hline UNB & Brazil & Brasília, DF & Centro Oeste \\
\hline UNESP & Brazil & São Paulo & Sudeste \\
\hline UNESP/FCAV & Brazil & Jaboticabal, SP & Sudeste \\
\hline UNICAMP & Brazil & Campinas, SP & Sudeste \\
\hline $\begin{array}{l}\text { UNIVERSIDADE DO ESTADO DE MATO } \\
\text { GROSSO }\end{array}$ & Brazil & Cáceres, MT & Centro Oeste \\
\hline UNIVERSIDADE DO ESTADO DO PARÁ & Brazil & Belém, PA & Norte \\
\hline UNIVERSIDADE DO VALE DO SÃO FRANCISCO & Brazil & Petrolina, PE & Nordeste \\
\hline $\begin{array}{l}\text { UNIVERSIDADE ESTADUAL DE PONTA } \\
\text { GROSSA }\end{array}$ & Brazil & Ponta Grossa, PR & Sul \\
\hline UNIVERSIDADE FEDERAL DA PARAÍBA & Brazil & João Pessoa, PB & Nordeste \\
\hline UNIVERSIDADE FEDERAL DE GOIÁS & Brazil & Goiânia, GO & Centro Oeste \\
\hline UNIVERSIDADE FEDERAL DE LAVRAS & Brazil & Lavras, MG & Sudeste \\
\hline UNIVERSIDADE FEDERAL DE PERNAMBUCO & Brazil & Recife, PE & Nordeste \\
\hline UNIVERSIDADE FEDERAL DE VIÇOSA & Brazil & Viçosa, MG & Sudeste \\
\hline UNIVERSIDADE FEDERAL DO CEARÁ & Brazil & Fortaleza, CE & Nordeste \\
\hline $\begin{array}{l}\text { UNIVERSIDADE FEDERAL DO ESPÍRITO } \\
\text { SANTO }\end{array}$ & Brazil & Vitória, ES & Sudeste \\
\hline UNIVERSIDADE FEDERAL DO MATO GROSSO & Brazil & Cuiabá, MT & Centro Oeste \\
\hline UNIVERSIDADE FEDERAL DO OESTE DO PARÁ & Brazil & Santarém, PA & Norte \\
\hline UNIVERSIDADE FEDERAL DO PARANÁ & Brazil & Curitiba, PR & Sul \\
\hline UNIVERSIDADE FEDERAL DO PARÁ & Brazil & Belém, PA & Norte \\
\hline UNIVERSIDADE FEDERAL DO PIAUÍ & Brazil & Teresina, PI & Nordeste \\
\hline UNIVERSIDADE FEDERAL FLUMINENSE & Brazil & Niterói, RJ & Sudeste \\
\hline UNIVERSIDADE RURAL DA AMAZÔNIA & Brazil & Belém, PA & Norte \\
\hline UNIVERSITAT MUNCHEN & Germany & Munique & Europa \\
\hline UNIVERSITY COPENHAGEN & Dinamarca & Copenhague & Europa \\
\hline UNIVERSITY LA MOLINA & Peru & La Molina & América do Sul \\
\hline UNIVERSITY OF ARIZONA & EUA & Tucson, AZ & EUA \\
\hline UNIVERSITY OF CAMBRIDGE & England & Cambridge & Europa \\
\hline UNIVERSITY OF COLORADO & EUA & Boulder & EUA \\
\hline UNIVERSITY OF EXETER & England & Exeter & Europa \\
\hline UNIVERSITY OF KANSAS & EUA & Lawrence, KS & EUA \\
\hline
\end{tabular}




\begin{tabular}{|c|c|c|c|}
\hline UNIVERSITY OF MARYLAND & EUA & College Park, MD & EUA \\
\hline UNIVERSITY OF NEW HAMPHSIRE & EUA & Durham, NH & EUA \\
\hline UNIVERSITY OF TARTU & Estônia & Tartu & Europa \\
\hline UNIVERSITY OF TOR VERGATA & Italy & Roma & Europa \\
\hline UPE & Brazil & Pernambuco & Nordeste \\
\hline URCAMP & Brazil & $\begin{array}{l}\text { Rio Grande do } \\
\text { Sul }\end{array}$ & Sul \\
\hline USFS (US FOREST SERVICE) & EUA & Washington, DC & EUA \\
\hline USP & Brazil & São Paulo & Sudeste \\
\hline UTAH STATE UNIVERSITY & EUA & Logan, UT & EUA \\
\hline UNB & Brazil & Brasília, DF & Centro Oeste \\
\hline UNICAMP & Brazil & São Paulo & Sudeste \\
\hline UNIVERSIDAD VEIGA DE ALMEIDA & Brazil & Cabo Frio, RJ & Sudeste \\
\hline UNIVERSIDADE ESTADUAL PAULISTA & Brazil & Araraquara, SP & Sudeste \\
\hline UNIVERSITE FRANÇOIS-RABELAIS DE TOURS & France & Tours & Europa \\
\hline UNIVERSITY PARK & EUA & Texas & EUA \\
\hline UNIVERSITY OF CALIFORNIA & EUA & Califórnia & EUA \\
\hline UNIVERSITY OF FLORIDA & EUA & Gainesville, FL & EUA \\
\hline UNIVERSITY OF HELSINKI & Finland & Helsinque & Europa \\
\hline UNIVERSITY OF LEEDS & England & Leeds & Europa \\
\hline UNIVERSITY OF NEW HAMPSHIRE & EUA & Durham, NH & EUA \\
\hline UNIVERSITY OF NOTTINGHAM & England & Nottingham & Europa \\
\hline UNIVERSITY OF OXFORD & England & Oxford & Europa \\
\hline UNIVERSITY OF VERMONT & EUA & Burlington, VT & EUA \\
\hline UTAH STATE UNIVERSITY & EUA & Logan, UT & EUA \\
\hline WESTERN MICHIGAN UNIVERSITY & EUA & Kalamazoo, MI & EUA \\
\hline WOODS HOLE RESEARCH CENTER & EUA & Falmouth, MA & EUA \\
\hline WAGENINGEN UNIVERSITY & Holanda & Wageningen & Europa \\
\hline ZHEJIANG A\&F UNIVERSITY & China & Zhejiang & Ásia \\
\hline INSTITUTO NACIONAL DO SEMIÁRIDO & Brazil & $\begin{array}{l}\text { Campina Grande, } \\
\text { PB }\end{array}$ & Nordeste \\
\hline
\end{tabular}

\section{$\rightarrow$ Impacto das Parcerias}

Num estudo sobre o impacto ${ }^{11}$ dessas parcerias, utilizando o software Publish or Perish, a Universidade de São Paulo (USP) -- em grande parte por meio da da Escola Superior de Agricultura Luiz de Queiroz (Esalq) -- foi a instituição nacional de maior relevância para a Unidade, com um total de 615 citações, para um conjunto de 24 trabalhos.

\footnotetext{
${ }^{11}$ A medida de impacto foi calculada pelo número de artigos produzidos em parcerias pela quantidade de citações obtidas no período de 2010 a 2015. O levantamento do número de parceiros foi obtido a partir do campo "afiliação" Ainfo.
} 
Em relação às instituições internacionais, a Indiana University foi a instituição com maior impacto, seguida do Departamento de Agricultura dos EUA (USDA). A primeira com 756 citações, para 18 artigos e a segunda com 451 citações, para 12 artigos. A relevância média, que pode ser vista na Tabela 7, entre o número de artigos pelo quantitativo de citações permite aferir uma maior importância à Indiana University, no que se refere ao impacto na comunidade científica, mas é preciso explorar essa análise situando-a no contexto de atuação da atual gestão e Agenda de pesquisas da Unidade.

Tabela 7 - Principais parceiros e relevância media

\begin{tabular}{|c|c|c|c|}
\hline Parceiro & Qtd. artigos & Citações & Relevância Média \\
\hline USP & 24 & 615 & 25,62 \\
\hline Indiana University & 18 & 756 & 42 \\
\hline USDA & 12 & 451 & 37,58 \\
\hline
\end{tabular}

A Tabela $8^{12}$ traz os principais artigos, citações e medidas de relevância pelos parceiros Indiana University, USDA e USP. Assim, são listados na mesma, os artigos produzidos pela Unidade em parceria com as três instituições, com informações a respeito do impacto de cada trabalho publicado pela Unidade e esses parceiros.

Tabela 8 - Principais artigos, citações e medidas de relevância pelos parceiros: Indiana University, USDA e USP

\section{Indiana University}

\section{Referência}

LU, D.; LI, G.; MORAN, E.; DUTRA, L.; BATISTELLA, M. A comparison of multisensor integration methods for land cover classification in the Brazilian Amazon. GIScience \& Remote Sensing, v. 48, n. 3, p. 345-370, 2011.

LU, D.; CHEN, Q.; WANG, G.; MORAN, E.; BATISTELLA, M.; ZHANG, M.; LAURIN, G. V.; SAAH, D. Aboveground forest biomass estimation with Landsat and LiDAR data and uncertainty analysis of the estimates. International Journal of Forestry Research, v. 2012. p. 16, 201216 p.

LU, D.; BATISTELLA, M.; MORAN, E.; HETRICK, S.; ALVES, D.;

\section{GS Cites}


BRONDIZIO, E. Fractional forest cover mapping in the Brazilian Amazon with a combination of MODIS and TM images. International Journal of Remote Sensing, v. 32, n. 22, p. 7131-7149, 2011.

LU, D.; LI, G.; BATISTELLA, M.; FREITAS, C. C. Mapping impervious surfaces with the integrated use of Landsat Thematic Mapper and radar data: a case study in an urban-rural landscape in the Brazilian Amazon. ISPRS Journal of Photogrammetry and Remote Sensing, v. 66, p. 798-808, 2011.

LI, G.; LU, D.; DUTRA, L.; BATISTELLA, M. A comparative analysis of ALOS PALSAR L-band and RADARSAT-2 C-band data for land-cover classification in a tropical moist region. ISPRS Journal of Photogrammetry and Remote Sensing, v. 70, p. 26-38, 2012. p. 26-38.

LU, D.; BATISTELLA, M.; LI, G.; MORAN, E.; HETRICK, S.; FREITAS, C.

DA C.; SANT'ANNA, S. J. Land use/cover classification in the Brazilian Amazon using satellite images. Pesquisa Agropecuária Brasileira, Brasilia, DF, v. 47 , n. 9 , p. $1185-1208$, set. 2012. p. 1185-1208.

LU, D.; BATISTELLA, M.; MORAN, E.; MAUSEL, P. Application of spectral mixture analysis to Amazonian land-use and land-cover classification. International Journal of Remote Sensing, v. 25, n. 23, p. 5345-5358, 2004.

LU, D.; LI, G.; VALLADARES, G. S.; BATISTELLA, M. Mapping soil erosion risk in Rondônia, Brazilian Amazonia: using rusle, remote sensing and GIS. Land Degradation \& Development, v. 15, p. 499-512, 2004.

LU, D.; BATISTELLA, M.; MORAN, E. Multitemporal spectral mixture analysis for Amazonian land-cover change detection. Canadian Journal of Remote Sensing, v. 30, n. 1, p. 87-100, 2004.

LU, D.; BATISTELLA, M.; MIRANDA, E. E. de. A comparative study of Não landsat TM and SPOT HRG images of vegetation classification in the brazilian Amazon. Photogrammetric Engineering \& Remote Sensing, v. 74, n. 3, p. 311localizado 321, mar. 2008. v. 74

LU, D.; BATISTELLA, M.; MORAN, E. Integration of landsat TM and SPOT HRG Images for vegetation change detection in the Brazilian Amazon. Photogrammetric Engineering \& Remote Sensing, v. 74, n. 4, p. 421-430, 2008.

LU, D.; BATISTELLA, M.; MORAN, E. Land-cover classification in the Brazilian Amazon with the integration of Landsat ETM + and Radarsat data. International Journal of Remote Sensing, v. 28, n. 24, p. 5447-5459, 2007.

LU, D.; BATISTELLA, M.; MAUSEL, P.; MORAN, E. Mapping and 46 monitoring land degradation risks in the western Brazilian Amazon using multitemporal landsat TM/ETM + IMAGES. Land Degradation \& Development, v. 18, p. 41-54, 2007.

LU, D.; BATISTELLA, M. Exploring TM image texture and its relationships 65 with biomass estimation in Rondônia, Brazilian Amazon. Acta Amazônica, v. 
35 , n. 2, p. 249-257, 2005. folhas avulsas

BATISTELLA, M.; MORAN, E. F. Dimensões humanas do uso e cobertura das terras na Amazônia: uma contribuição do LBA. Acta Amazônica, v. 35, n. 2, p. 239-247, 2005. folhas avulsas

LU, D.; BATISTELLA, M.; MORAN, E. Satellite estimation of aboveground biomass and impacts of forest stand structure. Photogrammetric Engineering and Remote Sensing, v. 71, n. 8, aug. 2005. p. 967-974. folhas avulsas

LU, D.; MAUSEL, P.; BATISTELLA, M.; MORAN, E. Land-cover binary

change detection methods for use in the moist tropical region of the Amazon: a comparative study. International Journal of Remote Sensing, v. 26, n. 1, jan. 2005, p. 101-114. 14 p. folhas avulsas

LU, D.; MAUSEL, P.; BATISTELLA, M.; MORAN, E. Comparison of land98 cover classification methods in the Brazilian Amazon Basin. Photogrammetric Engineering \& Remote Sensing, v. 70, n. 6, p. 723-731, jun. 2004.

\section{USDA}

\section{Referência}

DAVIDSON, E. A.; ARAUJO, A. C. de; ARTAXO, P.; BALCH, J. K.; BROWN, I.

F.; BUSTAMANTE, M. M. C.; COE, M. T.; DEFRIES, R. S.; KELLER, M.; LONGO, M.; MUNGER, J. W.; SCHROEDER, W.; SOARES-FILHO, B. S.; SOUZA JUNIOR, C. M.; WOFSY, S. C. The Amazon basin in transition. Nature, v. 481, p. 321-328, Jan. 2012.

HUNTER, M. O.; KELLER, M.; VICTORIA, D. de C.; MORTON, D. C. Tree height and tropical forest biomass estimation. Biogeosciences, v. 10, p. 10491-10529, 2013. p. 10491-10529.

STARK, S. C.; LEITOLD, V.; WU, J. L.; HUNTER, M. O.; CASTILHO, C. V. de; COSTA, F. R. C.; MCMAHON, S. M.; PARKER, G. G.; SHIMABUKURO, M. T.; LEFSKY, M. A.; KELLER, M.; ALVES, L. F.; SCHIETTI, J.; SHIMABUKURO, Y. E.; BRANDÃO, D. O.; WOODCOCK, T. K.; HIGUCHI, N.; CAMARGO, P. B. DE; OLIVEIRA JUNIOR, R. C. de; SALESKA, S. R. Amazon forest carbon dynamics predicted by profiles of canopy leaf area and light environment. Ecology Letters, v. 15, n. 12, dez. 2012. p. 1406-1414. Artigo publicado por Pesquisador Visitante da Embrapa Monitoramento por Satélite.

KAO, R. H.; GIBSON, C. M.; GALLERY, R. E.; MEIER, C. L.; BARNETT, D. T.; DOCHERTY, K. M.; BLEVINS, K. K.; TRAVERS, P. D.; AZUAJE, E.; SPRINGER, Y. P.; THIBAULT, K. M.; MCKENZIE, V. J.; KELLER, M.; ALVES, L. F.; HINCKLEY, E-L. S.; PARNELL, J.; SCHIMEL, D. NEON terrestrial field observations: designing continental-scale, standardized sampling. Ecosphere, v. 32, n. 
12, p. 1-17, dez. 2012. p. 1-17

SAATCHI, S.; MASCARO, J.; XU, L.; KELLER, M.; YANG, Y.; DUFFY, P.;

ESPÍRITO-SANTO, F.; BACCINI, A.; CHAMBERS, J.; SCHIMEL, D. Seeing the forest beyond the trees. Global Ecology and Biogeography, v. 23, n. 11, p. 1-5, nov. 2014. Artigo publicado por Pesquisador Visitante da Embrapa Monitoramento por Satélite.

ESPÍRITO-SANTO, F. D. B.; GLOOR, M.; KELLER, M.; MALHI, Y.; SAATCHI, S.; NELSON, B.; OLIVEIRA JUNIOR, R. C.; PEREIRA, C.; LLOYD, J.;

FROLKING, S.; PALACE, M.; SHIMABUKURO, Y. E.; DUARTE, V.;

MONTEAGUDO MENDOZA, A.; LÓPEZ-GONZÁLEZ, G.; BAKER, T. R.;

FELDPAUSCH, T. R.; BRIENEN, R. J. W.; ASNER, G. P.; BOYD, D. S.;

PHILLIPS, O. L. Size and frequency of natural forest disturbances and the Amazon forest carbon balance. Nature Communications, v. 5, art. n. 3434, 18 Mar. 2014.

SCHIMEL, D.; KELLER, M. Big questions, big science: meeting the challenges of global ecology. Oecologia, v. 177, n. 4, 2015. p 925-934.

ESPÍRITO-SANTO, F. D. B.; KELLER, M. M.; LINDER, E.; OLIVEIRA JUNIOR, R. C. de; PEREIRA, C.; OLIVEIRA, C. G. Gap formation and carbon cycling in the Brazilian Amazon: measurement using high-resolution optical remote sensing and studies in large forest plots. Plant Ecology \& Diversity, v. 16, n. 2, p. 25-46, abr./jun. 2013. Artigo publicado por Pesquisador Visitante da Embrapa Monitoramento por Satélite.

ANDERSEN, H. E.; REUTEBUCH, S. E.; MCGAUGHEY, R. J.; OLIVEIRA, M. V. N. D.; KELLER, M. Monitoring selective logging in western Amazonia with repeat lidar flights. Remote Sensing of Environment, v. 11, n. 3, p. 5783-5804, 2013.

SCHIMEL, D.; KELLER, M. Big questions, big science: meeting the challenges of global ecology. Oecologia, v. 177, n. 4, 2015. p 925-934.

HUNTER, M. O.; KELLE, M.; MORTON, D.; COOK, B.; LEFSKY, M.; DUCEY, M.; SALESKA, S.; OLIVEIRA JUNIOR, R. C. de; SCHIETTI, J. Structural dynamics of tropical moist forest gaps. Plos One, v. 10, n.7, p. 1-19, jul. 2015.

LEITOLD, V.; KELLER, M.; MORTON, D. C.; COOK, B. D.; SHIMABUKURO, Y. E. Airborne lidar-based estimates of tropical forest structure in complex terrain: opportunities and trade-offs for REDD+. Carbon Balance and Management, v. 10, n. 3, p. 1-12, 2015.

TOTAL

USP

Referência

DAVIDSON, E. A.; ARAUJO, A. C. de; ARTAXO, P.; BALCH, J. K.; BROWN, I.

F.; BUSTAMANTE, M. M. C.; COE, M. T.; DEFRIES, R. S.; KELLER, M.; 
LONGO, M.; MUNGER, J. W.; SCHROEDER, W.; SOARES-FILHO, B. S.; SOUZA JUNIOR, C. M.; WOFSY, S. C. The Amazon basin in transition. Nature, v. 481, p. 321-328, Jan. 2012.

NOGUEIRA, S. F.; PEREIRA, B. F. F.; GOMES, T. M.; DE PAULA, A. M.;

SANTOS, J. A. DOS; MONTES, C. R. Treated sewage effluent: Agronomical and economical aspects on bermudagrass production. Agricultural Water Management, v. 116, p. 151-159, 2012.

PEZZINI, F. F.; MELO, P. H. A. DE; OLIVEIRA, D. M. S. DE; AMORIM, R. X. DE; FIGUEIREDO, F. O. G. DE; DRUCKER, D. P.; RODRIGUES, F. R. DE O.; ZUQUIM, G.; EMILIO, T.; COSTA, F. R. C.; MAGNUSSON, W. E.; SAMPAIO, A. F.; LIMA, A. P.; GARCIA, A. R. DE. M.; MANZATTO, A. G.; NOGUEIRA, A.; COSTA, C. P. DA; BARBOSA, C. E. DE A.; BERNARDES, D.; CASTILHO, C. V. de; CUNHA, C. N. DA; FREITAS, C. G. DE; CAVALCANTE, C. DE O.; BRANDÃO, D. O.; RODRIGUES, D. DE J.; SANTOS, E. C. DA P. R. DOS; BACCARO, F. B.; ISHIDA, F. Y.; CARVALHO, F. A.; MOULATLET, G. M.; GUILLAUMET, J-L. B.; PINTO, J. L. P. V.; SCHIETTI, J.; VALE, J. D. DO; BELGER, L.; VERDADE, L. M.; PANSONATO, M. P.; NASCIMENTO, M. T.; SANTOS, M. C. V. DOS; CUNHA, M. S. DA; ARRUDA, R.; BARBOSA, R. I.; ROMERO, R. L.; PANSINI, S.; PIMENTEL, T. P. The Brazilian Program for Biodiversity Research (PPBio) Information System. Biodiversity \& Ecology, Hamburg, v. 4, p. 265-274, 2012. p. 265-274

VAN HAREN, J.; OLIVEIRA JUNIOR, R. C. de; BELDINI, P. T.; CAMARGO, P. B.; KELLER, M.; SALESKA, S. Tree species effects on soil properties and greenhouse gas fluxes in East-central Amazonia: comparison between Monoculture and Diverse Forest. Biotropica, v. 45, n. 6, p. 709-718, 2013. Artigo publicado por Pesquisador Visitante da Embrapa Monitoramento por Satélite.

DEMATTÊ, J. A. M.; TERRA, F. DA S.; QUARTAROLI, C. F. Spectral behavior of some modal soil profiles from São Paulo State, Brazil. Bragantia, Campinas, v. 71, n. 3, p. 413-423, 2012.

GUEDES FILHO, O.; VIEIRA, S. R.; CHIBA, M. K.; GREGO, C. R. Geostatistical analysis of crop yield maps in a long term no tillage system. Bragantia, Campinas, v. 69, suplemento, p. 9-18, 2010.

NOGUEIRA, S. F.; PAULA, A. M. de; PEREIRA, B. F. F.; SALEMI, L. F.; FONSECA, A. F. da; MONTES, C. R.; VICTORIA, R. L. Water type and irrigation time effects on microbial metabolism of a soil cultivated with Bermuda-grass Tifton 85. Brazilian Archives of Biology and Technology, v. 54, n. 3, p. 477-486, may/jun. 2011.

BUSTILlO, V.; VICTORIA, R. L.; MOURA, J. M. S. DE; VICTORIA, D. de C.; TOLEDO, A. M. A.; COLLICHIO, E. Factors driving the biogeochemical budget of the Amazon River and its statical modelling. Comptes Rendus Geoscience, v. 343, n. 4, p. 261-277, 2011.

BUSTILLO, V.; VICTORIA, R. L.; MOURA, J. M. S. de; VICTORIA, D. de C.; 
COLICCHIO, E. Biogeochemistry of carbon in the Amazonian Floodplains over a 2000-km reach: insights from a process-based model. Earth Interactions, Washington, v. 15, n. 4 , p. $1-29,2011$.

BUSTILLO, V.; VICTORIA, R. L.; MOURA, J. M. S. de; VICTORIA, D. de C.; TOLEDO, A. M. A.; COLLICCHIO, E. Biogeochemistry of the Amazonian floodplains insights from six end member mixing models. Earth Interactions, Washington, v. 14, n. 9, p. 1-83, 2010.

LIU, J.; HULL, V.; BATISTELLA, M.; DEFRIES, R.; DIETZ, T.; FU, F.; HERTEL, T. W.; IZAURRALDE, R. C.; LAMBIN, E. F.; LI, S.; MARTINELLI, L. A.; MCCONNELL, W. J.; MORAN, E. F.; NAYLOR, R.; OUYANG, Z.; POLENSKE, K. R.; REENBERG, A.; ROCHA, G. DE M.; SIMMONS, C. S.; VERBURG, P. H.; VITOUSEK, P. M.; ZHANG, F.; ZHU, C. Framing sustainability in a telecoupled world. Ecology and Society, v. 18, n. 2, jun. 2013. 19 p.

STARK, S. C.; LEITOLD, V.; WU, J. L.; HUNTER, M. O.; CASTILHO, C. V. de; COSTA, F. R. C.; MCMAHON, S. M.; PARKER, G. G.; SHIMABUKURO, M. T.; LEFSKY, M. A.; KELLER, M.; ALVES, L. F.; SCHIETTI, J.; SHIMABUKURO, Y. E.; BRANDÃO, D. O.; WOODCOCK, T. K.; HIGUCHI, N.; CAMARGO, P. B. DE; OLIVEIRA JUNIOR, R. C. de; SALESKA, S. R. Amazon forest carbon dynamics predicted by profiles of canopy leaf area and light environment. Ecology Letters, v. 15, n. 12, dez. 2012. p. 1406-1414. Artigo publicado por Pesquisador Visitante da Embrapa Monitoramento por Satélite.

PEREIRA, B. F. F.; GOMES, T. M.; NOGUEIRA, S. F.; MONTES, C. R.; MELFI, A. J. Cápsula porosa: interferência na atmosfera da solução do solo e metodologia de lavagem. Irriga, Botucatu, v. 14, n. 4, p. 441-448, out./dez. 2009.

TEIXEIRA, A. H. de C.; TONIETTO, J.; PEREIRA, G. E.; HERNANDEZ, F. B. T. Characterization of the Wine Grape Thermohydrological Conditions in the Tropical Brazilian Growing Region: Long-Term and Future Assessments ISRN Agronomy, v. 2014, p. 1-14, 2014.

PEREIRA, B. F. F.; HE, Z. L.; HERPIN, U.; NOGUEIRA, S. F.; MONTES, C. R.; MELFI, A. J. Reclaimed wastewater: impact on soil-plant system under tropical conditions. Journal of Hazardous Materials, v. 192, p. 54-61, 2011.

FRANCESCHINI, M. H. D.; DEMATTÊ, J. A. M.; SATO, M. V.; VICENTE, L. E.; GREGO, C. R. Abordagens semiquantitativa e quantitativa na avaliação da textura do solo por espectroscopia de reflectância bidirecional no VIS-NIR-SWIR. Pesquisa Agropecuária Brasileira, v. 48, n. 12, p. 1568-1581, dez. 2013.

GARDNER, T. A.; FERREIRA, J.; BARLOW, J.; LEES, A. C.; PARRY, L.;

VIEIRA, I. C. G.; BERENGUER, E.; ABRAMOVAY, R.; ALEIXO, A.; ANDRETTI, C.; ARAGÃO, L. E. O. C.; ARAÚJO, I.; ÁVILA, W. S. de;

BARDGETT, R. D.; BATISTELLA, M.; BEGOTTI, R. A.; BELDINI, T.; BLAS, D. E. de; BRAGA, R. F.; BRAGA, D. de L.; BRITO, J. G. de; CAMARGO, P. B. de; SANTOS, F. C. dos; OLIVEIRA, V. C. de; CORDEIRO, A. C. N.; CARDOSO, T. M.; CARVALHO, D. R. de; CASTELANI, S. A.; CHAUL, J. C. M.; CERRI, C. E.; 
COSTA, F. de A.; COSTA, C. D. F. da; COUDEL, E.; COUTINHO, A. C.; CUNHA, D.; D'ANTONA, A.; DEZINCOURT, J.; DIAS-SILVA, K.; DURIGAN, M.; ESQUERDO, J. C. D. M.; FERES, J.; FERRAZ, S. F. de B.; FERREIRA, A. E. de M.; FIORINI, A. C.; SILVA, L. V. F. da; FRAZÃO, F. S.; GARRETT, R.; GOMES, A. dos S.; GONÇALVES, K. da S.; GUERRERO, J. B.; HAMADA, N.; HUGHES, R. M.; IGLIORI, D. C.; JESUS, E. da C.; JUEN, L.; MIÉRCIO JUNIOR; OLIVEIRA JUNIOR, J. M. B. de; OLIVEIRA JUNIOR, R. C. de; SOUZA JUNIOR, C.; KAUFMANN, P.; KORASAKI, V.; LEAL, C. G.; LEITÃO, R.; LIMA, N.; ALMEIDA, M. de F. L.; LOURIVAL, R.; LOUZADA, J.; NALLY, R. M.; MARCHAND, S.; MAUES, M. M.; MOREIRA, F. M. S.; MORSELLO, C.; MOURA, N.; NESSIMIAN, J.; NUNES, S.; OLIVEIRA, V. H. F.; PARDINI, R.; PEREIRA, H. C.; POMPEU, P. S.; RIBAS, C. R.; ROSSETTI, F.; SCHMIDT, F. A.; SILVA, R. da; SILVA, R. C. V. M. da; SILVA, T. F. M. R. da; SILVEIRA, J.; SIQUEIRA, J. V.; CARVALHO, T. S. de; SOLAR, R. R. C.; TANCREDI, N. S. H.; THOMSON, J. R.; TORRES, P. C.; VAZ-DE-MELLO, F. Z.; VEIGA, R. C. S.; VENTURIERI, A.; VIANA, C.; WEINHOLD, D.; ZANETTI, R.; ZUANON, J. A social and ecological assessment of tropical land uses at multiple scales: the Sustainable Amazon Network. Philosophical Transactions of The Royal Society B, London, v. 368, n. 1619, p. 1-11, Apr. 2013.

SCHIETTI, J.; EMILIO, T.; RENNÓ, C. D.; DRUCKER, D. P.; COSTA, F. R. C.; NOGUEIRA, A.; BACCARO, F. B.; FIGUEIREDO, F.; CASTILHO, C. V. de; KINUPP, V.; GUILLAUMET, J-L.; GARCIA, A. R. M.; LIMA, A. P.; MAGNUSSON, W. E. Vertical distance from drainage drives floristic composition changes in an Amazonian rainforest. Plant Ecology \& Diversity, v. 8, n. 5, p. 1-13, 2013.

MATTOS, S. H. V. L. DE; PIQUEIRA, J. R. C.; VICENTE, L. E.; PEREZ FILHO, A. Criticalidade auto-organizada no cerrado? Invariância escalar dos padrões texturais e espectrais de fitosionomias do cerrado paulista. Revista Geonorte, Manaus, v. 1, n. 4, p. 149-161, 2012. Edição especial

BOUMANSS, R.; AMBRÓSIO, L. A.; ROMEIRO, A. R.; GODDI, E. M.;

FASIABEN, M. do C. R.; ANDRADE, D. C.; TOSTO, S. G.; MORAES, J. F. L. de; CAMARGO, L. A. S.; SINISGALLI, P. A. de A.; SOUSA JÚNIOR, W. C. de. Modelagem dinâmica do uso e cobertura das terras para o controle da erosão na bacia hidrográfica do rio Mogi-Guaçú e Pardo - São Paulo - Brasil. Revista Iberoamericana de Economía Ecológica, Bellaterra, v. 14, p. 1-12, 2010.

ANGELOCCI, L. R.; VILLA NOVA, N. A.; COELHO FILHO, M. A.; MARIN, F. $\mathrm{R}$. Measurements of net radiation absorbed by isolated acid lime trees (Citrus latifolia Tanaka). Journal of Horticultural Science \& Biotechnology, v. 79, n. 5, p. 699-703, 2004.

ANGELOCCI, L. R.; MARIN, F. R.; OLIVEIRA, R. F. DE; RIGHI, E. Z.

Transpiration, leaf diffusive conductance, and atmospheric water demand relationship in an irrigated acid lime orchard. Brazilian Journal of Plant Physiology, Campinas, v. 16, n. 1, p. 53-64, 2004.

OLIVEIRA JUNIOR, R. C. de; KELLER, M. M.; RAMOS, J. F. da F.; BELDINI, T. 
P.; CRILL, P. M.; CAMARGO, P. B. de; HAREN, J. van. Chemical analysis of rainfall and throughfall in the Tapajós National Forest, Belterra, Pará, Brazil. Revista Ambiente \& Água, v. 10, n. 2, p. 263-285, abr./jun. 2015.

FRANCESCHINI, M. H. D.; DEMATÊ, J. A. M.; VICENTE, L. E.;

BARTHOLOMEUS, H.; SOUZA FILHO, C. R. DE. Prediction of soil properties

using imaging spectroscopy: Considering fractional vegetation cover to improve accuracy. International Journal of Applied Earth Observation and Geoinformation, v. 38, p. 358-370, 2015.

Salienta-se que os parceiros são agentes estratégicos para a construção do conhecimento da Unidade. Entender o impacto dessas contribuições pode fornecer condições para identificar em quais e com quais podemos nos relacionar, quando o objetivo maior for o impacto.

\section{CONSIDERAÇÕES FINAIS}

Criar mecanismos para sistematizar o intercâmbio entre conhecimento disponibilizados por pesquisadores e suas pesquisas aos usuários das soluções tecnológicas é um desafio, por vezes, complexo. Nesse sentido, é importante experimentar formas distintas para estruturar ações, tendo a organização de informações como uma ação estratégica para auxiliar a área PD\&I, na elaboração e adequação de seus resultados de pesquisa.

Este estudo tentou trazer novas oportunidades instrumentais para o processo de análise da produção técnico-científica das Unidades da Embrapa, conduzido pela Secretaria de Gestão e Desenvolvimento Institucional (SDI), uma vez que apresentou a base Google Scholar como uma plataforma possível para utilização nesse processo. Nesse aspecto, verificou-se que a Web of Science apresentou percentual inferior ao da base Google Scholar, para o número de artigos em periódicos produzidos pela Embrapa Territorial. Assim, por não evidenciar o número real de artigos produzidos e, consequentemente, o impacto da produção da Unidade, medido pelo número de citações, pode influenciar negativamente a Unidade avaliada.

Ressalta-se que o artigo não teve como finalidade qualificar artigos, ou seja, determinar qualificação a partir de comparativos de classificação que é realizada pelos órgãos 
competentes. Considera-se como um estudo inicial apresentado em uma prerrogativa de que se fazem necessários esforços para clareza de onde estão sendo dirigidos os esforços das pesquisas na instituição.

Ao final, constata-se que os resultados alcançados aqui, contribuem no subsídio de indicadores das Unidades. Para efeitos de avaliação institucional, recomenda-se que a aplicação desse tipo de estudo ocorra periodicamente, de modo a subsidiar a Embrapa em suas decisões estratégicas.

Sabe-se que a quantidade de citação em bases de referências é uma questão privilegiada dentro da instituição. Assim viabilizar outros meios de obtenção de como essa produção é medida, se faz necessário. É relevante ampliar as fontes de estudo, uma vez que bases, como Scopus e Scielo, oferecem serviços robustos para análise bibliométrica e cientométricas. Torna-se oportuno ainda estudá-las para ver de forma individual o desempenho de cada Unidade da Embrapa. Somado a isso, torna-se estratégico entender o impacto das áreas de atuação da Unidade, a partir da produção total, o que não foi perseguido neste artigo.

Neste raciocínio, se empreendermos esforços nas pesquisas de como está ocorrendo o fenômeno desde o ponto da publicação à sua repercussão, estará de acordo com o que se propõe a Embrapa no que se refere à disponibilização dos resultados de pesquisa à sociedade. 


\section{REFERÊNCIAS}

ALVES, E. J.; GONÇALVES, C. A.; BAX, M. P. Métodos ágeis sob a ótica da informação. Informação \& Informação, Londrina, v. 22, n. 3, 2017. Disponível em: http://www.brapci.inf.br/v/a/28397. Acesso em: 22 Fev. 2018.

ANTUNES, J. F. G.; OLIVEIRA, S. R. de M. Ainfo: a experiência da Embrapa na disponibilização e recuperação de informação. Ciência da Informação, Brasília, DF, v. 27, n. 1, 1998. Disponível em: http://www.scielo.br/scielo.php?script=sci_arttext\&pid=S010019651998000100011\&lng=en\&nrm=iso. Acesso em: 30 out. 2019.

FALAGAS, W. E.; PTSOUNI, E. I.; MALIETZIS, G. A.; PAPPAS, G. Comparison of PubMed, Scopus, Web of Science, and Google Scholar: strengths and weaknesses. The FASEB Journal, Bethesda, MD, v. 22, n. 2, p. 338-342, 2007.

LIMA, R. A. de; VELHO, L. M. L. S.; FARIA, L. I. L. de. Bibliometria e "avaliação" da atividade científica: um estudo sobre o índice h. Perspectivas em Ciência da Informação, Belo Horizonte, 2012, v. 17, n. 3, p. 03-17. Disponível em: http://dx.doi.org/10.1590/S141399362012000300002. Acesso em: 30 out. 2019.

MADRUGA, S. N.; GAMBA, C. M.; TRINDADE, M. O Google Scholar como instrumento disseminador da produção intelectual da Faculdade de Medicina Veterinária e Zootecnia da Universidade de São Paulo - FMVZ/USP. RBBD. Revista Brasileira de Biblioteconomia e Documentação, São Paulo, v. 11, p. 365-373, maio 2016. Disponível em: https://rbbd.febab.org.br/rbbd/article/view/516. Acesso em: 06 nov. 2019.

NASCIMENTO, A. G. Altmetria para bibliotecários: guia prático de métricas alternativas para avaliação da produção científica. São Paulo: Scortecci, 2016. 148 p.

PENTEADO FILHO, R. de C.; FONSECA JUNIOR, W. C. da; AVILA, A. F. D. Perfil da produção de artigos da Embrapa entre 2007 e 2015: oportunidades e desafios. Brasília, DF: Embrapa, 2017. 90 p. (Embrapa. Secretaria de Gestão e Desenvolvimento Institucional, 17).

PEREIRA, C. A.; FUJINO, A. Cartografia dos estudos métricos da informação: contribuições para qualificação da avaliação da ciência. In: In: ENCONTRO NACIONAL DE PESQUISA EM CIÊNCIA DA INFORMAÇÃO (ENANCIB), 16., 2015, João Pessoa-PB. Anais... Belo Horizonte, João Pessoa-PB: Universidade Federal da Paraíba.

PINTO, D. M.; SOLANO, V. de O.; SANTOS, V. V. dos. Embrapa Monitoramento por satélite: um estudo a partir de periódicos indexados. In: ENCONTRO BRASILEIRO DE BIBLIOMETRIA E CIENTOMETRIA, 5., 2016, São Paulo. Anais... São Paulo: USP, 2016. p. Disponível em: https://ainfo.cnptia.embrapa.br/digital/bitstream/item/145406/1/4740.pdf. Acesso em: 22 fev. 2018.

SANDES-GUIMARÃES, L. V. Impacto. RAE Publicações, São Paulo: FGV, 2016. Disponível em: http://rae.fgv.br/manual-rae/impacto. Acesso em: 26 fev. 2018. 
SANTOS, R. N. M. dos.; KOBASHI, N. Y. Aspectos metodológicos da produção de indicadores em ciência e tecnologia. In: ENCONTRO NACIONAL DE ENSINO E PESQUISA EM INFORMAÇÃO, 6., 2005, Salvador. Anais... Salvador: UFBA, 2005. Disponível em: http://www.ufpe.br/ppgci/images/publicacoesdocentes/raimundo/04.pdf . Acesso em: 30 out. 2019. 